Changing a urine collection pad (UCP) every 30 minutes reduced contamination of urine samples more than a UCP kept in the nappy in children with suspected urinary tract infection

Rao S, Bhatt J, Houghton C, et al. An improved urine collection pad method: a randomised clinical trial. Arch Dis Child 2004;89:7735 .

Is a urine collection pad (UCP) that is replaced every 30 minutes better than a UCP kept in the nappy (diaper) for reducing bacterial contamination of urine samples to rule out suspected urinary tract infection (UTI) in children?

METHODS

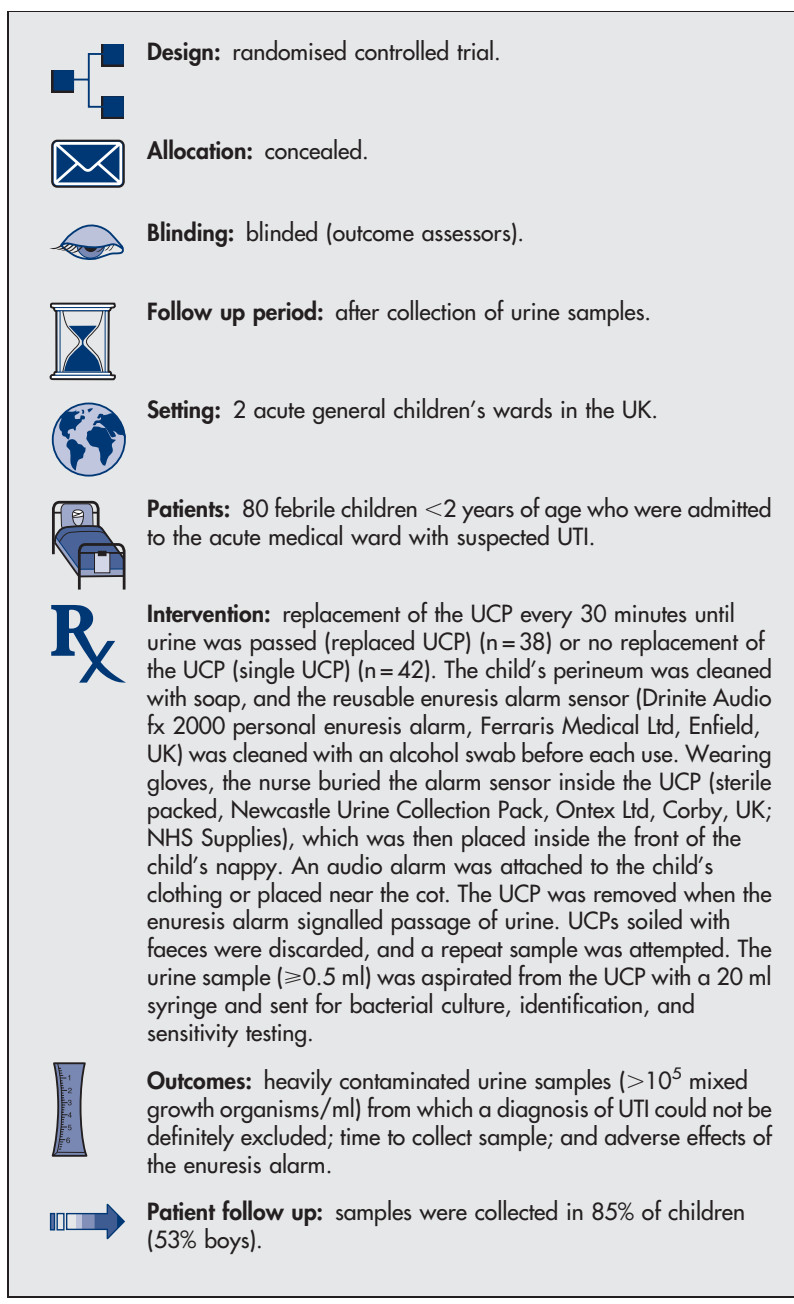

For correspondence: Dr P Macfarlane, Rotherham General Hospital Rotherham, UK. peter.macfarlane@rothgen.nhs.uk

Source of funding: no external funding.

\section{MAIN RESULTS}

3 children $(4 \%)$ had a UTI $\left(>10^{5}\right.$ organisms/ml pure single organism growth). Children in the replaced UCP group had fewer contaminated urine samples than those in the single UCP group (table). The replaced and single UCP groups did not differ for time to collect samples $(\mathrm{n}=47$ [59\%], median $45 \vee 80 \mathrm{~min}, \mathrm{p}=0.056)$. No enuresis alarm adverse effects occurred.

\section{CONCLUSION}

A urine collection pad (UCP) that was replaced every 30 minutes was better than a UCP kept in the nappy for reducing bacterial contamination of urine samples from children with suspected urinary tract infection.

\section{Commentary}

ollection of uncontaminated urine samples from infants and young children has always presented a challenge for both nurses and parents. For this reason, the advent of the UCP is regarded as a welcome alternative to the unpopular and sometimes traumatic urine collection bag or the "time consuming and messy" clean catch method that has been used in the past.'

The randomised control trial by Rao et al not only confirms the value of UCPs for collecting urine from young children but also shows the advantages of changing pads every 30 minutes until urine is passed.

The strength of the study lies in the randomisation of children and the detailed description of the methods used to prepare the child and monitor for the passage of urine. An increased sample size and more even distribution of the sexes between the 2 groups would have added further rigour to the study and reduced the potential for bias reported by the authors. In addition, more information about the wait time for collection for each sex would have provided useful information.

Although the results of Rao et al clearly indicate a benefit of changing UCPs every 30 minutes, the feasibility of implementing these procedures in clinical settings remains open to debate. Only 11 of the 24 children in the replaced UCP group did not require a change of pad. 6 children, however, required 3-7 pad changes. Thus, because changing UCPs every 30 minutes may add to the workload of already busy nurses, the practical value of these findings could be viewed with ambivalence.

The most useful finding, however, may be that methods other than UCPs should be considered for urine collection in children with diarrhoea because faecal soiling was noted by Rao et al as a primary reason for UCP failure.

Jeanette Robertson, RN, MSc, FRCNA Women's and Children's Health Service Perth, Western Australia, Australia

1 Liaw LC, Nayar DM, Pedler SJ, et al. BMJ 2000;320:1312-3.

Urine collection pad (UCP) replacement every 30 minutes (replaced UCP) $v$ no replacement (single UCP) for collection of urine samples in children $<2$ y with suspected urinary tract infection*

\begin{tabular}{|c|c|c|c|c|}
\hline Outcome & Replaced UCP & Single UCP & RRR $(95 \% \mathrm{Cl})$ & NNT (Cl) \\
\hline 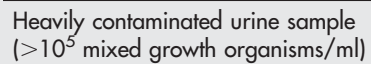 & $3 \%$ & $29 \%$ & $89 \%$ (38 to 98 ) & $4(3$ to 12$)$ \\
\hline
\end{tabular}

